\title{
Understanding human biological rhythm. Therapeutic principles of bright light and melatonin for sleep disorders of circadian rhythm
}

\section{Sunao Uchida', ${ }^{1,3}$ Masaki Nishida' ${ }^{2,3}$ Kohei Shioda', Yuko Morita'}

1. Faculty of Sport Sciences, Waseda University, Tokorozawa

2. Department of Neuropsychiatry, Tokyo Medical and Dental University, Tokyo

3. Future Institute for Sport Sciences, Waseda University, Tokyo

Indian J Sleep Med 2010; 5. 1, 8-12

\begin{abstract}
In this review, the authors explain how human circadian rhythm has developed in the process of evolution, mechanisms of human circadian control, modification of human circadian rhythm and therapeutic principles of circadian rhythm related sleep disorders. In order to treat circadian rhythm related sleep disorders, precise understanding of the mechanism and proper use of circadian phase modifiers are necessary. Therapeutic principles involve bright light, melatonin, exercise and their phase response curves.
\end{abstract}

\section{Introduction}

There are two aspects to sleep disorders. One is its quality, and the other its timing. Disorders such as insomnia, sleep breathing disorders, narcolepsy or sleep related movement disorders manifest with disturbed sleep quality. Circadian rhythm sleep disorders (ICSD-2: see BOX 1), on the other hand, do not necessarily have sleep quality problem. For example, patients with delayed sleep phase syndrome usually have normal sleep quality when they are able to sleep soundly. Rather their problem is from "a malalignment between the timing of the individual's circadian rhythm of sleep propensity and 24-hour social and physical environments (ICSD-2)”. In this article, the authors explain the basic biological mechanism and therapeutic options for circadian rhythm disorders.

\section{Address for correspondence}

\section{Sunao Uchida}

Faculty of Sport Sciences, Waseda University

2-579-15 Mikajima

Tokorozawa, Saitama 359-1192 JAPAN

Email:sunao@waseda.jp
BOX 1: ICSD-2 Circadian Rhythm Sleep Disorders

For optimal sleep, the desired sleep time should match the timing of the circadian rhythm of sleep and wake propensity. Therefore, a recurrent or chronic pattern of sleep disturbance may result from alterations of the circadian timing system or a misalignment between the timing of the individual's circadian rhythm of sleep propensity and 24-hour social and physical environments. These disorders may arise when the physical environment is altered relative to the internal circadian timing or the circadian timing system is altered relative to the external environment. In addition to physiological and environmental factors, maladaptive behaviors influence the presentation and severity of the circadian rhythm sleep disorders. A circadian rhythm sleep disorder is defined by the following criteria:

General Criteria for Circadian Rhythm Sleep Disorder

A. There is a persistent or recurrent pattern of sleep disturbance due primarily to one of the following:

i. Alterations of the circadian timekeeping system

ii. Misalignment between the endogenous circadian rhythm \& exogenous factors that affect the timing or duration of sleep.

B. The circadian related sleep disruption leads to insomnia, excessive daytime sleepiness, or both.

C. The sleep disturbance is associated with impairment of social, occupational, or other areas of functioning.

Classification

Delayed Sleep Phase Type Advanced Sleep Phase Type

Irregular Sleep-Wake Type Free Running Type

Jet Lag Type

(Non-entrained)

Shift Work Type Due to Medical Condition

Others : Due to Drug or Substance (eg: Alcohol) abuse 


\section{Evolution of circadian rhythm}

Our life cycle is a 24 hours cycle. This 24-hour cycle is very suitable for our daily life. Therefore, we usually are not even aware of it. However, if one thinks of the rotation period of other planets, it is easily understood that 24-hour is not a matter of course (Table 1). For example, the longest rotation period of a planet in the solar system is that of Venus, which is 243.02 days. Moreover, the rotation of Venus is in the opposite direction to that of Earth. So, if one happens to be on Venus, one will see the sun rising from the western horizon and the daylight length would be about 121.51 earth days (the length would also depend on the 'season'). It is believed that there are no biological organisms/life existing on Venus, but if there are "Venusians", they would be surprised to see how hurriedly we spend a day and night on the Earth!

Since the very first single-celled organism appeared, all the organisms on the Earth have been in an environment manifested by a 24-hour day night cycle. Therefore in the process of evolution, organisms adapted themselves to such an environment. This probably was the manner of evolution of the 24 hour biological rhythm (circadian rhythm). It should be noted that the sun light has had an essential role in the evolution process of the circadian rhythm.

\section{Center of human circadian rhythm}

It has been known that the center of human biological clock is located in the suprachiasmatic nucleus (SCN)

Table 1 : Time for rotation of planets (negative number indicate rotation for opposite direction from the earth. For more detailed information, see http://cseligman.com/ text/sky/rotationvsday.htm)

\begin{tabular}{|l|l|}
\hline Planet & Time for one rotation \\
\hline Mercury & 58.6467 days \\
\hline Venus & -243.02 days \\
\hline Earth & $23 \mathrm{hr} 56 \mathrm{~min} 4.1 \mathrm{sec}$ \\
\hline Mars & $24 \mathrm{hr} 37 \mathrm{~min} 22.66 \mathrm{sec}$ \\
\hline Jupiter & $9 \mathrm{hr} 55 \mathrm{~min} 30 \mathrm{sec}$ \\
\hline Saturn & $10 \mathrm{hr} 32 \mathrm{~min} 35 \mathrm{sec}$ \\
\hline Uranus & $-17 \mathrm{hr} 14 \mathrm{~min} 24 \mathrm{sec}$ \\
\hline Neptune & $16 \mathrm{hr} 6.6 \mathrm{~min}$ \\
\hline Pluto & -6 days $9 \mathrm{hr} 17.6 \mathrm{~min}$ \\
\hline
\end{tabular}

of the anterior hypothalamus. The intrinsic mechanism within a SCN cell has been well studied. There are positive and negative feedback loops within a cell that forms an intrinsic circadian clock oscillation (Reppert $\&$ Weaver, 2002). The SCN receives fibers directly from retina, which convey information of brightness. The light-dark cycle formed by rotation of the earth entrains SCN circadian rhythm to the 24 hour biological rhythm.

\section{How do we measure human circadian rhythm?}

The SCN also entrains slave oscillators in other parts of the body. In humans, two major physiological circadian correlates are usually used. One is core body temperature (Figure 1) and the other is serum (saliva, or urinary) melatonin concentration (Figure 2).

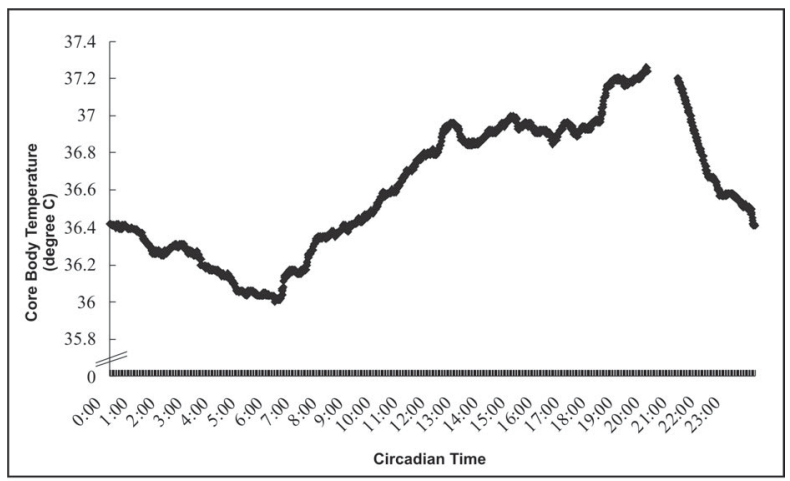

Figure 1: Circadian variation of body temperature.

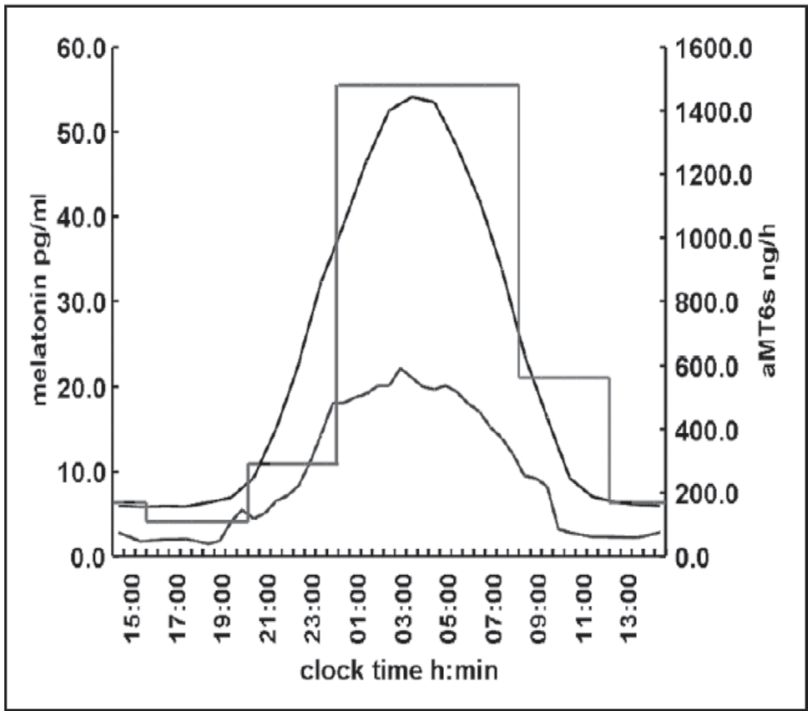

Figure 2: A figure from Prof Josephine Arendt's text. http:// www.endotext.org/neuroendo/neuroendo15/ch01s05. html Black line indicates serum, blue saliva and red urinary melatonin concentrations.

Indian Journal of Sleep Medicine (IJSM), Vol. 5, No. 1, 2010 
When human circadian rhythm is measured, the most frequently used physiological correlate is core body temperature. Core body temperature is usually measured using a probe inserted into the rectum. A standard device to measure rectal core body temperature is shown on figure 3 (probe type). Another device to measure core body temperature is a capsule type thermometer.

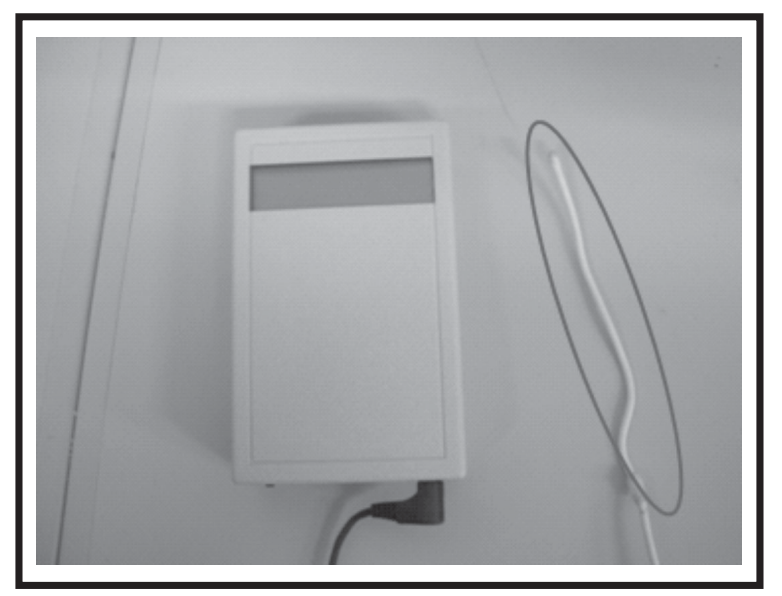

Figure 3: Device to measure rectal core body temperature. Circled part will be inserted into rectum through anus.

The device is designed to be swallowed. The capsule moves through digestive tract and stays in the body for 12 to 48 hours (capsule type). The advantage of the probe type thermometer is to be able to measure continuously for many days except during toilet use. Also, probe type thermometer measures core body temperature consistently in the same region, whereas the capsule type moves through the digestive tract. Advantage of the capsule type thermometer is its convenience. Whereas for temperature recording using a capsule type thermometer requires a light recording device, the rectal thermometer can be an uncomfortable experience. However as recordings have to be done over long periods of time more than one capsule is required.

Melatonin is an intrinsic substance secreted from the pineal gland located in the dorsal thalamus. Melatonin is secreted during the night and has a circadian pattern. Melatonin secretion is suppressed by bright light (Lewy, Sack and Singer 1985). Therefore, when one needs to precisely measure the melatonin secretion pattern, control of light conditions is necessary. Usually a dim light condition (about 100 lux) is used. By manipulation of the light conditions a clear secretion pattern is observed (Figure 2). The timing of increasing serum melatonin concentration is defined as dim light melatonin onset (DLMO). DLMO is an important marker in the circadian phase (Lewy and Sack, 1989).

\section{Phase response curves (PRC)}

In the above section, human circadian phase measurements have been explained. It is known that there are factors that could shift the human circadian phase. Bright light is the strongest factor to shift the circadian phase. The reason is that circadian rhythm itself was developed by the light dark cycle of the earth as noted in the earlier part of this review. A graph which shows the relationship between time of the day and phase shift effect (advance positive, delay negative) is called "phase response curve (PRC)".

A phase response curve to bright light has been reported (Khals et al. 2003) Figure 4 shows a PRC to bright light from a review article by Hashimoto et al (2007). The

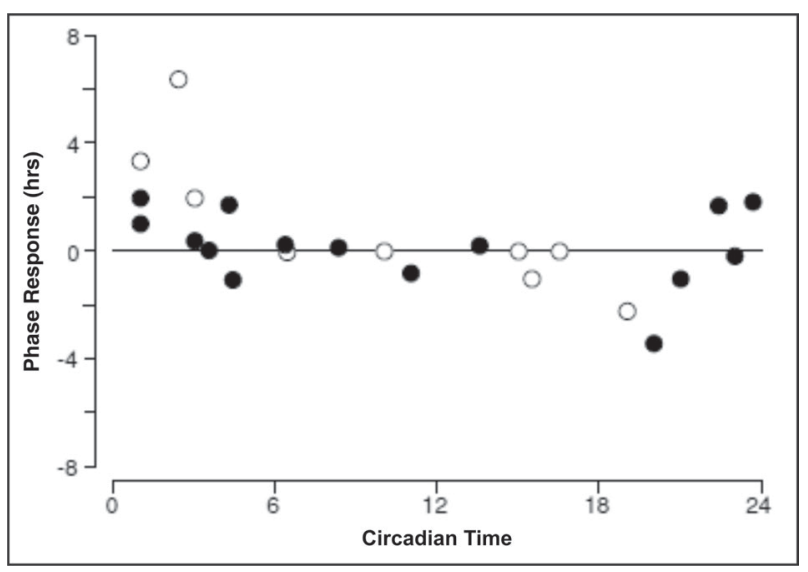

Figure 4: Phase response curve to the bright light. This figure is from Hashimoto S's review \& open circle is from Honma \&Honma. 1988 data, and black dots are from Minors, et al. 1991 data.

graph indicates that the strongest phase advance effect was obtained by morning bright light whereas evening bright light gives strongest phase delay effect.

PRC to oral melatonin administration has also been known. Oral melatonin administration also has a strong effect to circadian phase shift. Figure 5 (Zee and Manthena, 2007) shows the effect of oral melatonin administration and bright light on the PRC. The melatonin PRC shows almost opposite phase to bright light, as early morning melatonin administration has a phase delay effect. It has been considered low dose melatonin is sufficient enough for phase shift, since higher dose melatonin tends to remain in the body to the opposite phase effect time.

Bright light and melatonin are two important factors for circadian phase shift. However, there are other factors 


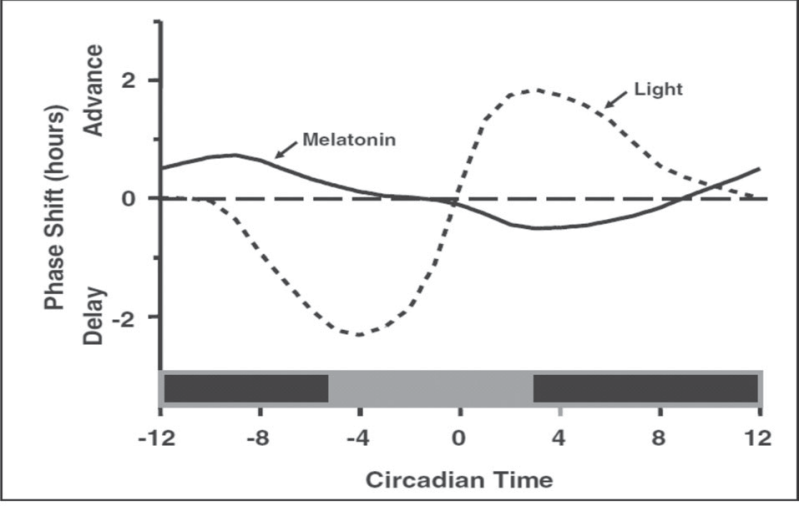

Figure 5: Phase response curve to Melatonin.

which shift the circadian phase; exercise being one of them. Figure 6 (Edwards et al. 2009) shows phase response curve to exercise. It has a similar phase response pattern to the bright light. The authors consider that it may because of the co-existing bright light effect, but at least night time phase delay would have contribution to phase delay.

As explained above, various factors have different phase shift effect of the human circadian phase. However, it could be considered that there is a general law to shift the circadian phase as shown on figure 7. Stimuli before

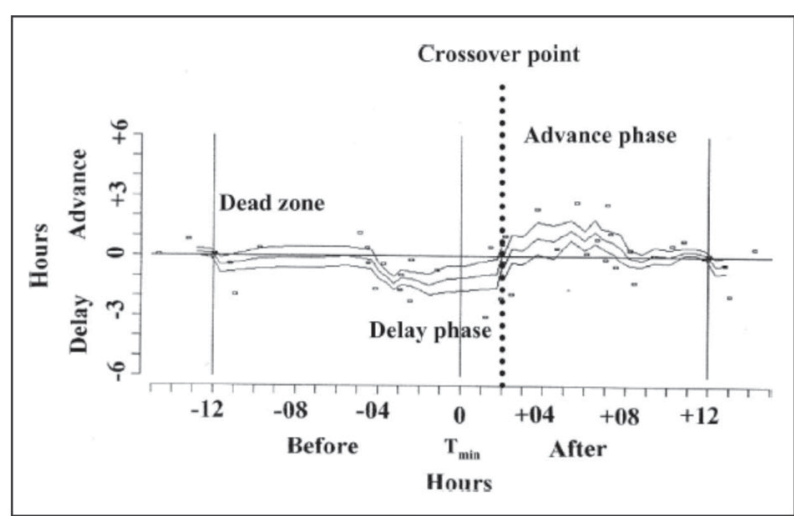

Figure 6: Phase response curve to Exercise.

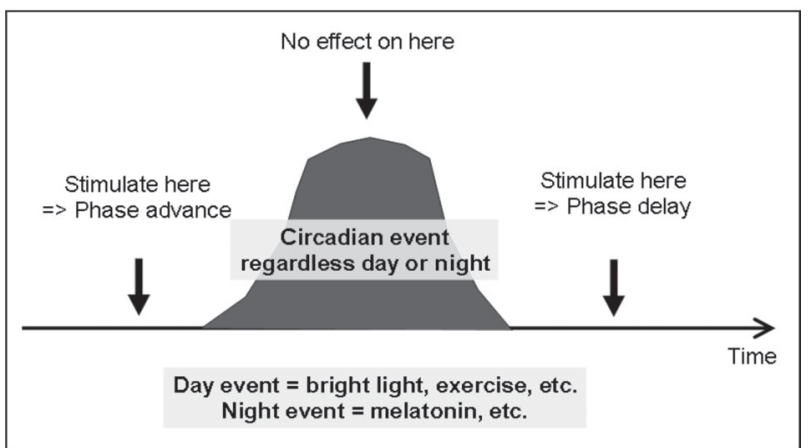

Figure 7: In general, stimulus before the event advance the circadian phase, while stimulus after the event delay the circadian phase. the circadian event time generally has phase advance effect and after the circadian event has phase delay effect. For example, bright light is a day time event and early morning bright light has a phase advance effect. On the other hand, melatonin secretion is a night time event and late afternoon melatonin administration (before melatonin secretion) has a phase advance effect. This general law could help in understanding better the phase response curve.

\section{Pathophysiology and treatment of delayed sleep phase syndrome}

There are four types circadian sleep disorders which are not related to forced alterations of the sleep-wake cycle or light-dark cycle. They are delayed sleep phase type, advanced sleep phase type, irregular sleep-wake type, and free running type (non-entrained) in ICSC-3. In this section, the pathophysiology and treatment strategy of most commonly observed delayed sleep phase type (DSPT) syndromes are explained.

The exact pathophysiology of DSPT is not known. However, it is generally believed that the abnormality of the circadian regulation mechanism is involved (Corwley et al. 2007). Sensitivity to light, which entrain circadian phase may be especially disturbed. Okawa and Uchiyama's group has done a detailed study of the circadian property of DSPT (Okawa and Uchiyama, 2007). In the semi-constant routine environment, DSPT patients showed larger interval between body temperature nadir and sleep off-set, and the BT nadir is significantly earlier than controls. Based on these results, they hypothesized that DSPT patients fail to be exposed to bright light while they are in the phase advance period of their PRC, as they are tend to be sleeping.

DSPT patients fail to wake up in the normal morning hours. Rather they start to sleep late in the night or early morning, (normally 3:00am - 7:00am) and sleep through the morning hours, sometimes until mid afternoon. One would think that such people could try to wake up earlier regardless of the sleep length, so that one could have a sleepy day but could sleep earlier (normal sleeping time). It usually does not work. Normal people sometimes stay up late at night until, for example, four o'clock in the morning and wake up seven o'clock. However, they do not have less problem waking up after short sleep than DSPT patients. It is because of the difference of sleep propensity phase (sleepy hours). After

Indian Journal of Sleep Medicine (IJSM), Vol. 5, No. 1, 2010 
a late night short sleep, normal people wake up when the sleep propensity phase terminate, whereas DSPT needs to wake up in the middle of sleep hours. Moreover, they need to stay up during the sleep phase in the morning hours (see figure 8 ). Therefore, the treatment of DSPT should not be just to allocate sleep time, but to shift their circadian phase. In order to shift the circadian phase, bright light and melatonin are the two strongest tools. The usage of bright light and melatonin have been explained in the previous sections.

\begin{tabular}{|c|c|c|}
\hline 12:00 18:00 & No:00 \\
\hline & Normal Sleep Phase \\
& Short Sleep \\
DSPS Sleep Hours \\
DSPS Sleep Phase \\
\hline
\end{tabular}

Figure 8: When normal sleepers stay awake longer and have short sleep, they wake up in normal hours, while DSPS patients have the short sleep, they need to wake up in the middle of their sleep phase.

\section{Summary}

- Human 24 hours circadian rhythm is a result of adaptation to rotation time of the earth.

- Human circadian rhythm is controlled by the suprachiasmatic nucleus in anterior hypothalamus.

- Some people have abnormal circadian phase or abnormal circadian time, which does not allow them to adapt themselves to social life.

- Bright light and melatonin could be used to adjust circadian rhythm.

- Such adjustment should be used in the right timing in the phase response curve.

\section{References}

The International Classification of SLEEP DISORDERS, Second Edition (ICSD-2), American Academy of Sleep Medicine, Westchester, IL. 2005.

Reppert, S.M., Weaver, D.R. Coordination of circadian timing in mammals. Nature 2002; 418(6901):935-941.

Lewy AJ, Sack RL \& Singer CM. Immediate and delayed effects of bright light on human melatonin production: Shifting 'dawn' and 'dusk' shifts the dim light melatonin onset (DLMO). Ann NYAcad Sci 1985; 453: 253-259.

Lewy AJ, Sack RL. The dim light melatonin onset as a marker for circadian phase position. Chronobiol Int. 1989; 6(1):93-102.

Khalsa SB, Jewett ME, Cajochen C, Czeisler CA. A phase response curve to single bright light pulses in human subjects. J Physiol. 2003 Jun 15; 549(Pt 3):945-52. Epub 2003; Apr 25.

Hashimoto S, Honma S, Honma K. Sleep and biological rhythms. Folia Pharmacol. Jpn. 2007; 129:400-403 [in Japanese]

Zee PC, Manthena P. The brain's master circadian clock: Implications and opportunities for therapy of sleep disorders. (2007) Sleep Medicine Reviews, 11(1):59-70

Edwards BJ, Reilly T, Waterhouse J. Zeitgeber-effects of exercise on human circadian rhythms: what are alternative approaches to investigating the existence of a phase-response curve to exercise? Biological Rhythm Research, 2009; 40(1):53-69

Crowley SJ, Acebo C, Carskadon MA. Sleep, circadian rhythms, and delayed phase in adolescence. Sleep Med 2007; 8:602-612.

Okawa M, Uchiyama M. Circadian rhythm sleep disorders: characteristics and entrainment pathology in delayed sleep phase and non-24-h sleep-wake syndrome. Sleep Med Rev. 2007 Dec; 11(6):485-96. 